\title{
Reduction of Thermal Strain Induced Rate Error for Navigation Grade Fiber Optic Gyroscope
}

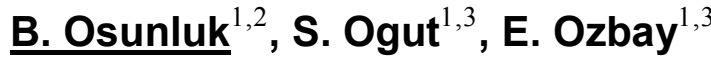 \\ ${ }^{1}$ Department of Electrical and Electronics Engineering \\ Bilkent University \\ 06800 Ankara \\ TURKEY \\ ${ }^{2}$ Microelectronics, Guidance and Electro-optics Sector \\ Aselsan Inc. \\ 06011 Ankara \\ TURKEY \\ ${ }^{3}$ Nanotechnology Research Center (NANOTAM) \\ Bilkent University \\ 06800 Ankara \\ TURKEY
}

Inertial Sensors and Systems 2018

Braunschweig, Germany 


\begin{abstract}
In this paper, we present the latest efforts of developing a navigation grade fiber optic gyroscope coil in Turkey. One of the main precision limits for a fiber optic gyroscope coil is its sensitivity to thermally induced rate error. We used an advanced thermal modeling of a fiber optic gyroscope coil in order to obtain the strain fields along the coil and calculate the rate error. An intermediate validation step which compares the strain field calculations with the optical time domain reflectometer (OTDR) measurements of a laboratory fiber optic gyroscope setup is presented. In literature, there are several works indicating the relation between strain and rate error and trying to reduce the total strain as a whole [1], [2]. The strain analysis is separated into axial and radial gradients along the fiber coil. A new fiber coil structure using titanium as the spool material is designed and two fiber coils are produced. Fiber coils are tested under a temperature profile ranging from $-40{ }^{\circ} \mathrm{C}$ to $+60{ }^{\circ} \mathrm{C}$. Simulation and experimental results are consistent. Proposed coil design is promising with 8-fold reduction in rate error.
\end{abstract}

\title{
1. Introduction
}

Fiber optic gyroscope (FOG) is a mature technology for high precision inertial navigation applications. The wide usage area of the technology brings the demand for better performance under environmental disturbances. One of the main performance limits is the thermally induced rate error. Most of the work in the literature propose to control the error by reducing the total stress along the fiber coil as a whole.

In this paper, we proposed an approach for stress analysis for a fiber coil. Although the total stress and strain fields do not change, rate error is reduced as stress homogeneity increases. Strain homogeneity measurement is not a straightforward application. We use finite element model (FEM) analysis to obtain the strain distribution along the fiber coil.

\section{Theory}

\subsection{Shupe Equation and Elastooptic Interactions}

Thermal fluctuations create nonreciprocal phase shift between counterpropagating waves in the fiber coil. Nonreciprocal phase shift is defined by Shupe [3], given in equation 1. Mohr [4] extends this analysis for elastooptical interactions (see equation 2). Temperature and stress change results phase shift on fiber segment $d s$ which is integrated along all the fiber coil. 


$$
\begin{aligned}
& \Delta \phi=\frac{\beta_{0}}{c} \int_{0}^{L}\left(\dot{n}+n \dot{\varepsilon_{z}}\right)(L-2 s) d s \\
& \dot{n}=-\frac{n^{3}}{2}\left[\left(p_{11}+p_{12}\right) \dot{\varepsilon_{r}}+p_{11} \dot{\varepsilon_{z}}\right]
\end{aligned}
$$

Here $\beta_{0}$ is the free space propagation constant, $\dot{n}$ is the refractive index change, $\dot{\varepsilon}_{z}$ and $\dot{\varepsilon}_{r}$ are the strain changes along z-direction (the direction light wave travels) and r-direction (radial direction of fiber core), $L$ is the total fiber length and $s$ is the distance from one of the fiber coil ends. In this equation $d s$ states the fiber segment which is three dimensional in space.

\subsection{Phase Error Calculation Approach}

We have developed a method for the calculation of the phase error. Fiber coil is a cylindrical structure and the integral can be represented in radius, azimuth and height $(r, \theta, z)$. Fiber coil consists of fiber core, cladding, coating and adhesive. Light travels only in the fiber core so integral is taken through fiber core which is only continuous through azimuth and discrete for axial and radial directions. We reduce the equation to two dimensions and discretize it for calculation.

$$
\Delta \phi(t)=\frac{\beta_{0}}{c} \sum_{i=1}^{N_{\text {radial }}} \sum_{j=1}^{N_{\text {axial }}} \int_{0}^{2 \pi}\left(\dot{n}\left(r_{i}, \theta, z_{j}, t\right)+n \dot{\varepsilon_{z}}\left(r_{i}, \theta, z_{j}, t\right)\right)(L-2 s) d \theta \Delta s
$$

Where $N_{\text {radial }}, N_{\text {axial }}$ are the radial and axial layer numbers and $N=N_{\text {radial }} \times N_{\text {axial }}$ is the total number of turns. Here radial and axial subscripts define the axial and radial directions of the fiber coil cross-section. Combining the Sagnac relation with the equation,

$$
\Omega(t)=\frac{n}{\pi N} \sum_{i=1}^{N_{\text {radial }}} \frac{1}{d_{i}^{2}} \sum_{j=1}^{N_{\text {axial }}} \int_{0}^{2 \pi}\left(\dot{n}\left(r_{i}, \theta, z_{j}, t\right)+n \dot{\varepsilon_{z}}\left(r_{i}, \theta, z_{j}, t\right)\right)(L-2 s) d \theta \Delta s
$$

Here $d_{i}$ is the diameter of each turn. Diameter of the fiber coil increases for each layer through radius. Using the relation $\Delta s=\frac{L}{N}=\frac{\pi d_{i} N}{N}=\pi d_{i}$, , and taking the integral over $d \theta$ results as

$$
\Omega(t)=\frac{n}{N} \sum_{i=1}^{N_{\text {radial }}} \frac{1}{d_{i}} \sum_{j=1}^{N_{\text {axial }}}\left(\dot{n}\left(r_{i}, z_{j}, t\right)+n \dot{\varepsilon_{z}}\left(r_{i}, z_{j}, t\right)\right)\left(L-2 s-l_{i}\right)
$$


where $l_{i}$ is the length of each fiber turn which is different for every radial layer. This equation is the 2-D approximation for calculation of the total phase error due to any disturbance (temperature, vibration, moisture...) that changes the strain field.

\section{Modeling of Fiber Coil}

In our previous work, we presented a model for thermally induced rate errors for a fiber optic gyroscope with quadrupole winding [5] (Figure 1). In that work, we have developed a simulation environment for calculation of the errors of a fiber coil design and verified the simulation environment with experimental results of three different fiber coils. We concluded that elastooptic effect is much stronger than the pure Shupe error for that coil design.
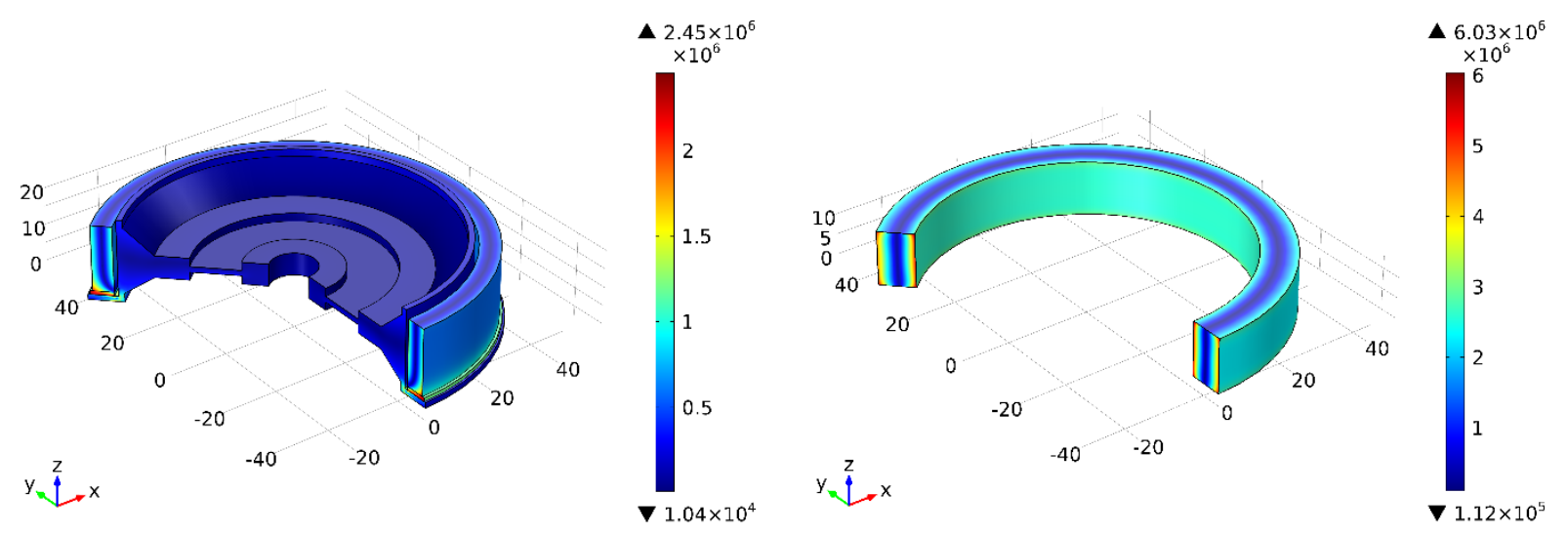

Figure 1. Finite element model simulation of the fiber coil with and without spool

The elastooptic effect is directly related to the stress-strain characteristics of the fiber coil. We run a simulation to obtain the total refractive index change and total elongation of a fiber coil under temperature variation. Refractive index change is found to be $7 \times 10^{-6}{ }^{\circ} \mathrm{C}^{-1}$ which is close to the temperature coefficient of refractive index for fiber core material, $10^{-5}{ }^{\circ} \mathrm{C}^{-1}$. Total strain change due to thermal variation is calculated and compared with OTDR measurements of a fiber coil.

\section{Strain Measurements}

Fiber coil is subjected to a temperature change from $-40{ }^{\circ} \mathrm{C}$ up to $+60{ }^{\circ} \mathrm{C}$ and the flight time inside the fiber coil is measured continuously with the OTDR. The flight time includes the fiber refractive index change due to temperature which is compensated by using the coefficient. OTDR measurement and the calculated strain values are given in Figure 2. Experimental and simulation results are consistent. 


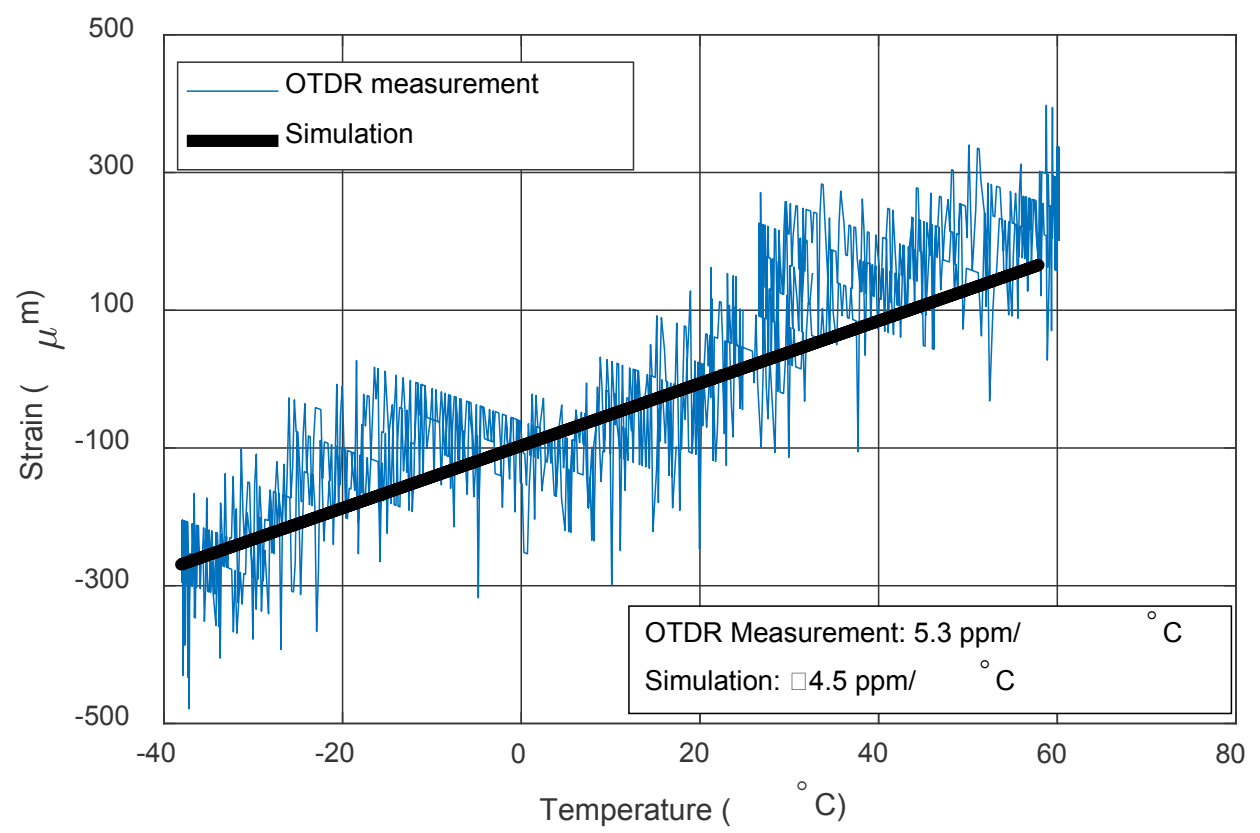

Figure 2. Comparison of OTDR measurement and simulation output

Consistent results provide an intermediate verification step for the strain calculations of our model. We use the verified model for further analysis.

\section{High Performance Fiber Coil Design}

In order to reduce the elastooptic effects, we designed a new fiber coil without spool and with titanium spool, which has a temperature expansion coefficient much closer to fiber. We obtained stress and strain values from the model. Figure 3 shows the Von Mises stress distribution along the fiber coil with the aluminum spool and Table 1 gives the stress values of newly designed fiber coils. Although Von Mises stress characteristics and values stay same, bias error significantly reduced. 


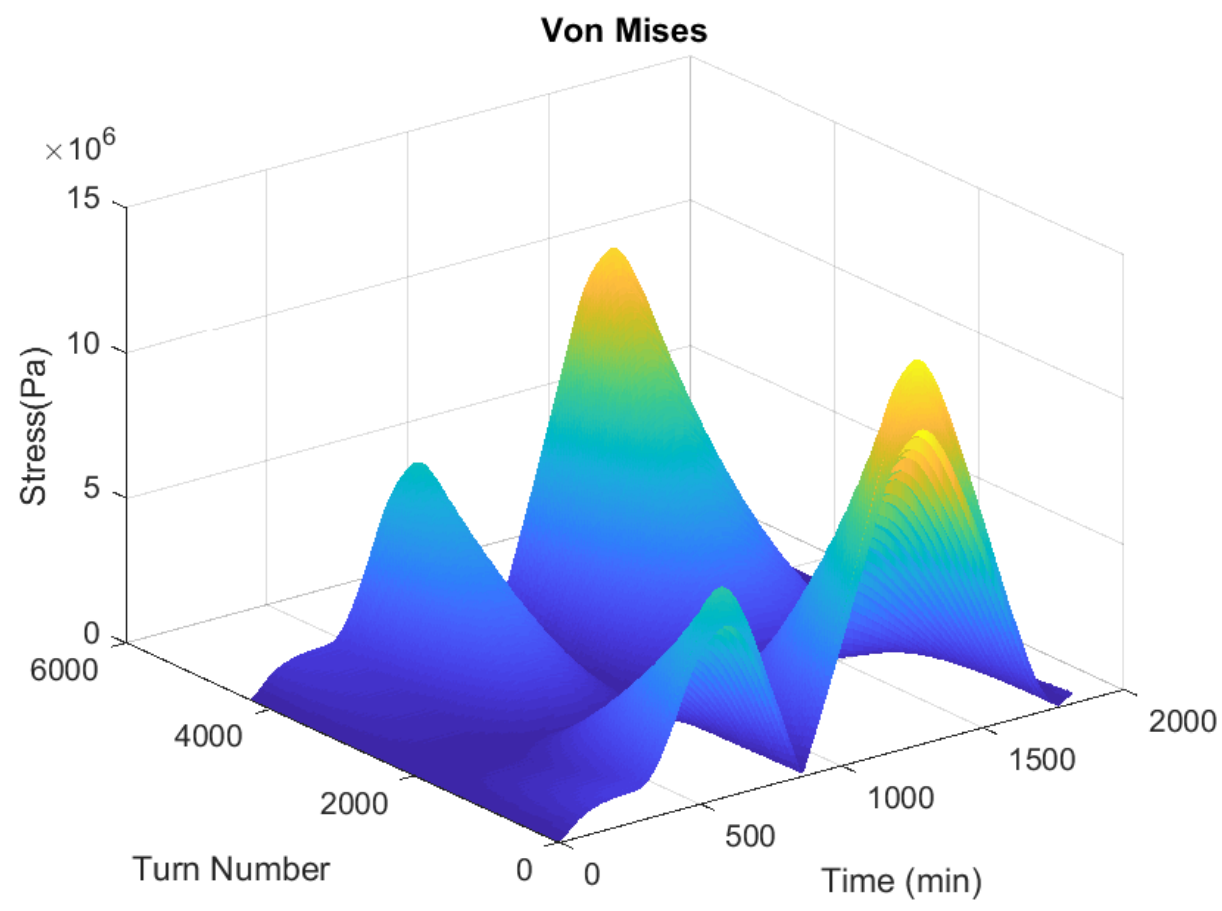

Figure 3. Stress distribution along the fiber coil while the temperature is changing

Table 1: Von Mises stress values for different spool materials

\begin{tabular}{|c|c|c|}
\hline Spool Material & $\begin{array}{c}\text { Maximum Von Mises } \\
\text { Stress (Pa) }\end{array}$ & $\begin{array}{c}\text { Average Von Mises } \\
\text { Stress (Pa) }\end{array}$ \\
\hline Aluminum & $1.34 \times 10^{7}$ & $2.15 \times 10^{6}$ \\
\hline Titanium & $1.32 \times 10^{7}$ & $2.14 \times 10^{6}$ \\
\hline No Spool & $1.31 \times 10^{7}$ & $2.14 \times 10^{6}$ \\
\hline
\end{tabular}

\subsection{Strain Inhomogeneity}

Equation 5 provides a basis for analysis of the strain gradients in axial and radial directions. We calculate the temperature coefficient of strain change for each fiber turn. Strain temperature coefficient versus fiber turn location is a serrated line, where serration indicates the axial strain and line indicates the radial strain (see Figure 4.). Although the change in the strain temperature coefficient is higher in the radial direction than the axial, practical quadrupole asymmetry for the Shupe coefficient is much higher for the axial direction. Our simulations show that the axial strain gradient is the dominant source of rate error for our fiber coil. 


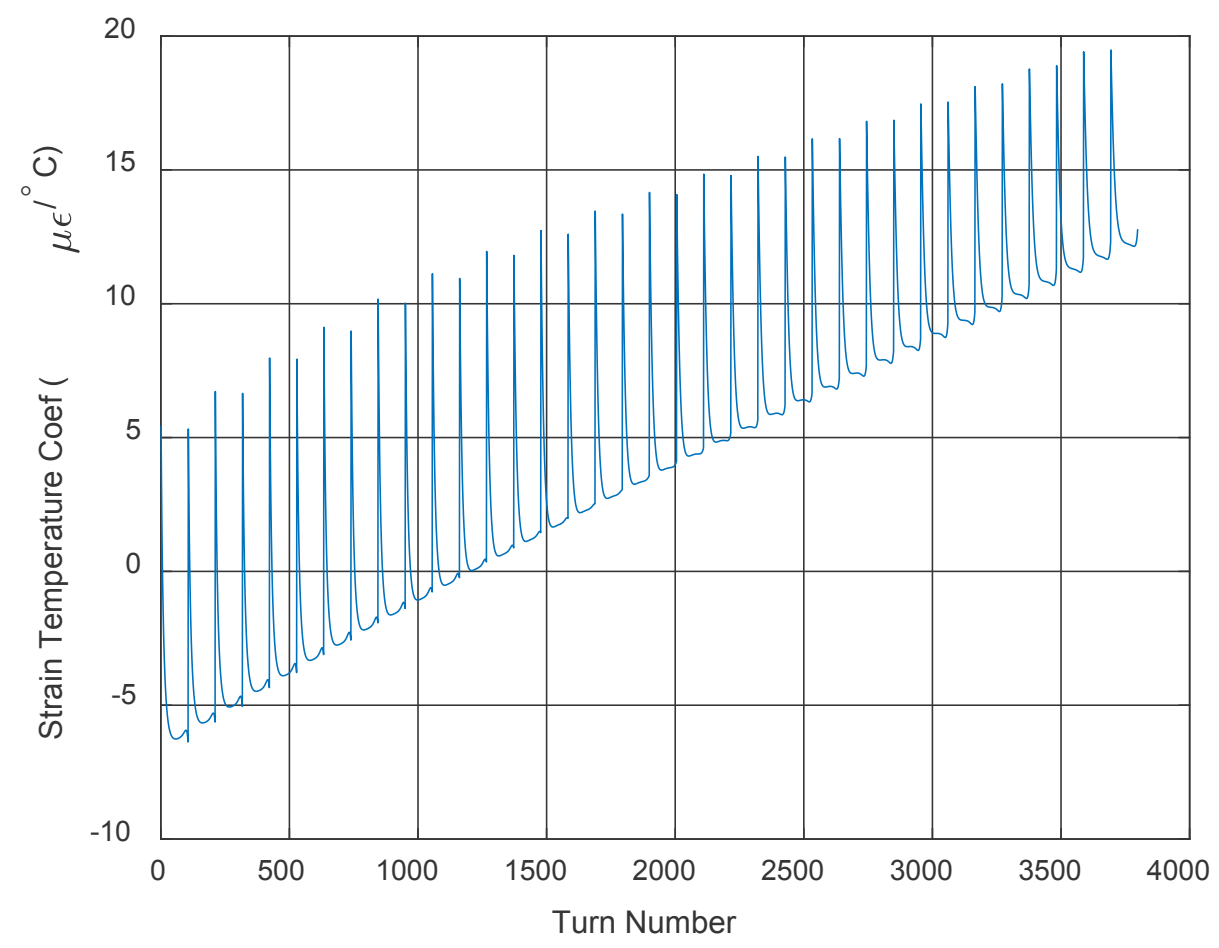

Figure 4. Strain temperature coefficient for each turn number

Strain coefficients of each turn in the same radius are averaged and named as axial strain coefficients. This gives the inhomogeneity in the fiber coil axial direction. The same method is carried out to obtain the radial strain coefficients. Calculated strain coefficients are given in Figure 5 for different spool materials. Radial strain temperature coefficient change is a straight line, while axial strain coefficient shows an asymmetric characteristic. Although the value of radial coefficient change is higher than the axial coefficient, the rate error of radial layers cancels out each other, thanks to quadrupole winding pattern.
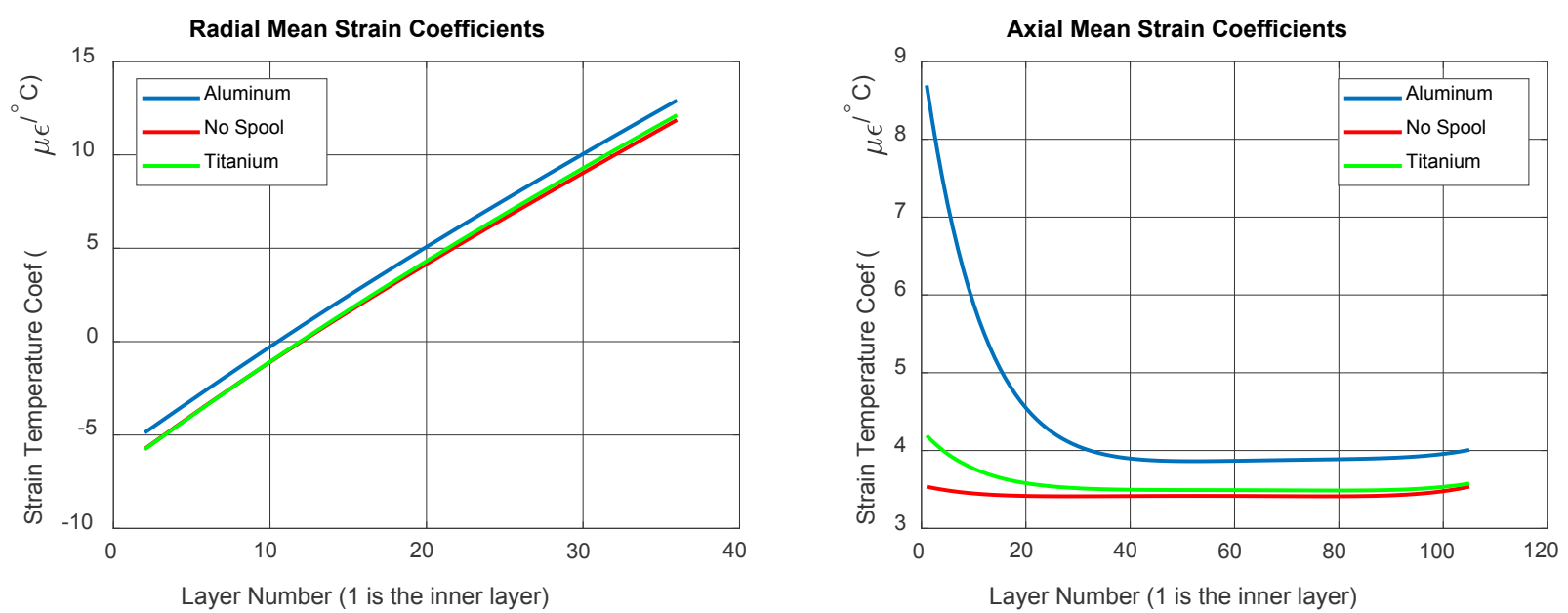

Figure 5. Strain temperature coefficients for different spool configurations 
Strain temperature coefficient change for axial direction is limited. However, it is the main contributor to phase error if the winding pattern has an axial asymmetry, as for the practical quadrupole pattern. It is also seen from Figure 5 that, radial strain coefficients do not differ too much for different spool configurations. Axial strain coefficient asymmetry is higher for aluminum spool than titanium spool and no spool configurations.

\subsection{Experiment}

A closed-loop fiber optic gyroscope setup is built for laboratory experiments. This setup consists of one ASE light source, two MIOC - fiber coil pairs, and digital and analog electronic boards. The setup is placed in a temperature chamber during temperature tests. Temperature sensors are mounted on each coil spool to monitor the coil temperature.
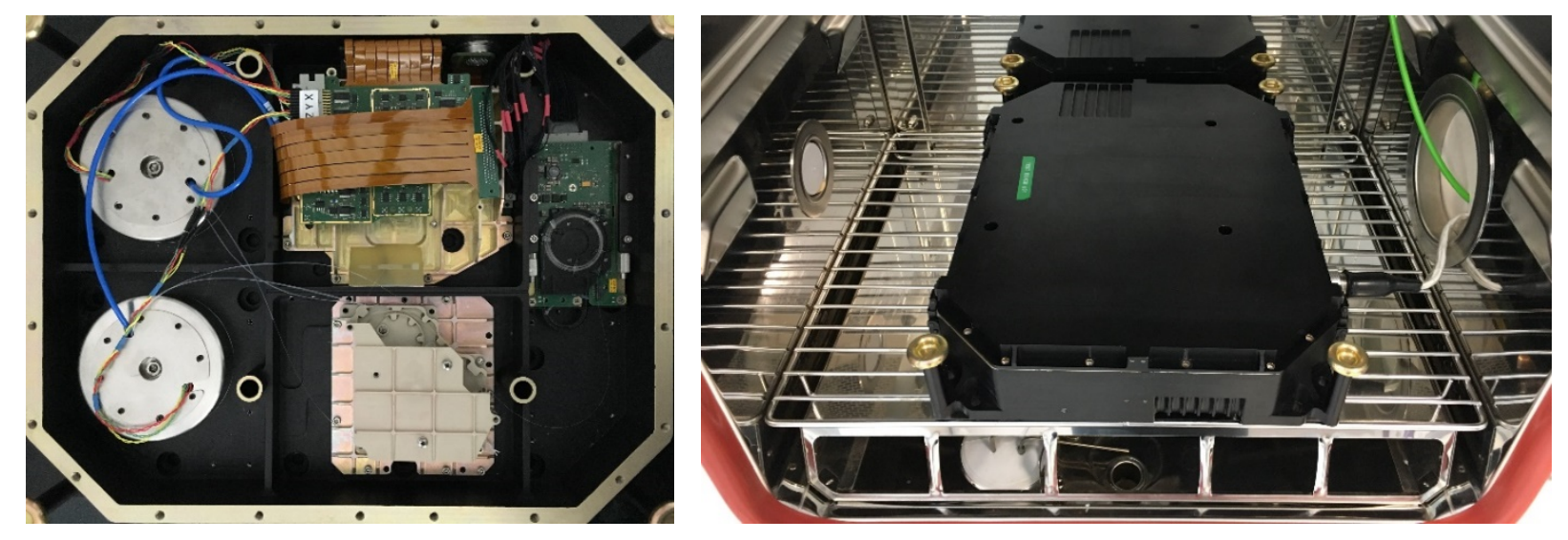

Figure 6. Setup for fiber coil temperature experiments

Temperature profile ranging from $-40^{\circ} \mathrm{C}$ to $+60^{\circ} \mathrm{C}$ is applied to fiber coils while temperature and rotation rate measurement data are collected. Collected rotation rate data is processed to eliminate the earth rotation and gyro noise. Experimental and simulation results are plotted in Figure 7 . Thermal sensitivity coefficient of a fiber coil is defined as the ratio of the rate error to the temperature derivative. Calculated coefficients are given in Table 2. 


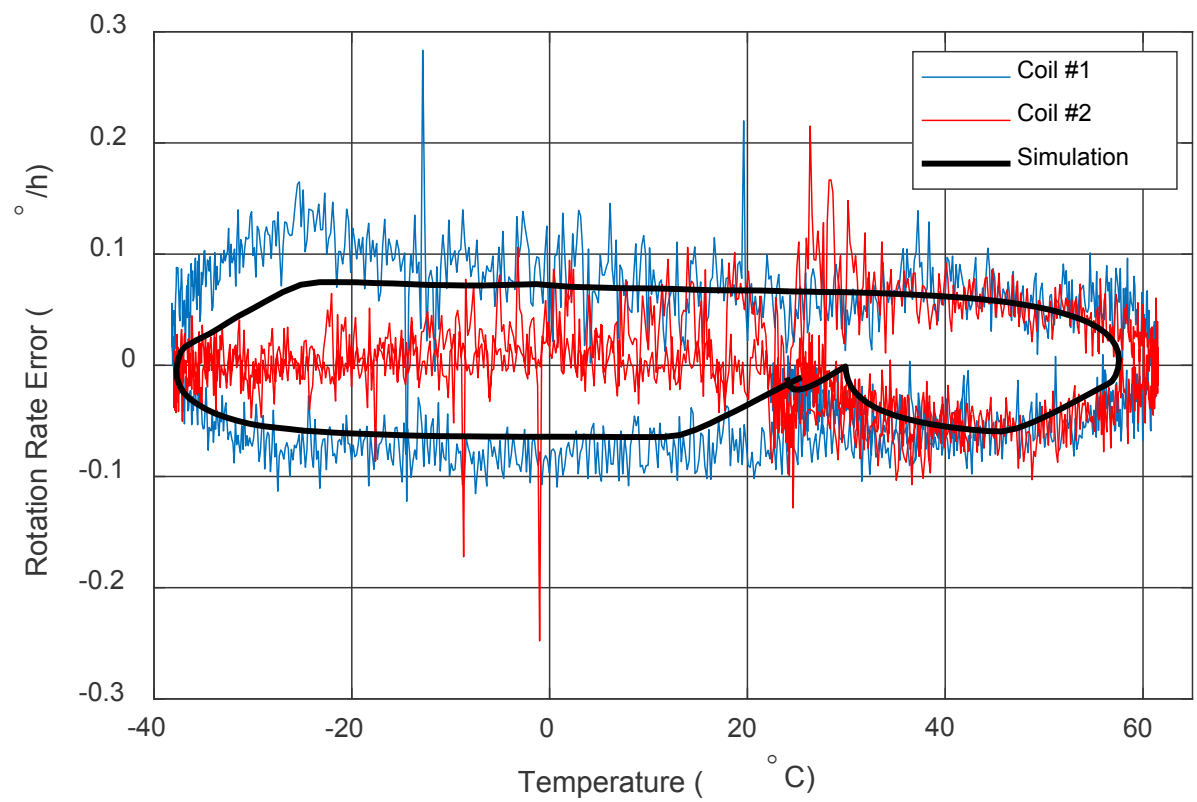

Figure 7: Experimental data for two fiber coils and simulation result of the coil design

Table 2: Thermal sensitivities of the fiber coils and the model

\begin{tabular}{|c|c|}
\hline Coil No & $\begin{array}{c}\text { Thermal Sensitivity } \\
\text { Coefficient } \\
\left(\frac{{ }^{\circ} / \boldsymbol{h}}{\left({ }^{\circ} \mathrm{C} / \mathbf{m i n}\right.}\right)\end{array}$ \\
\hline Coil \#1 & -0.41 \\
\hline Coil \#2 & -0.16 \\
\hline Simulation & -0.40 \\
\hline
\end{tabular}

Rate error characteristics of the two fiber coils are consistent with the theoretical model. Coil-1 is nearly perfectly matched with the simulation result. Coil-2 also aligned with the simulation result above room temperature. However, Coil-2 thermal sensitivity reduces nearly to zero below room temperature. This phenomenon is called the racket effect in the literature.

\section{Conclusion}

We showed strain inhomogeneity can result in rate error for a fiber coil. We designed a new coil by using the simulation model with better performance. We verified the model with laboratory experiments. 


\section{References}

[1] S. Minakuchi, et. al., "Thermal strain in lightweight composite fiber-optic gyroscope for space application", Journal of Lightwave Technology, vol. 33, no. 12, pp. 26582662, 2015.

[2] E. Quatraro, et. al., "High performance FOG for non temperature stabilized environment", 2011 DGON Inertial Sensors \& Systems, Germany, 2011

[3] D. M. Shupe, "Thermally induced non-reciprocity in the fiber optic interferometer," Applied Optics, vol. 19, no. 5, pp. 654-655, 1980.

[4] F. Mohr, "Bias error fiber optic gyroscopes due to elastooptic interactions in the sensor fiber," Proceedings of SPIE, vol. 5502, pp. 410-413, 2004.

[3] B. Osunluk, S. Ogut and E. Ozbay, "Thermally induced bias errors for a fiber coil with practical quadrupole winding", Proc. IEEE Inertial Sensors and Systems, pp. 152155, 2017 